\title{
Non-Fickian dispersive transport of strontium in laboratory-scale columns: Modelling and evaluation
}

DOI:

10.1016/j.jhydrol.2017.03.053

\section{Document Version}

Accepted author manuscript

Link to publication record in Manchester Research Explorer

\section{Citation for published version (APA):}

Liu, D., Jivkov, AP., Wang, L., Si, G., \& Yu, J. (2017). Non-Fickian dispersive transport of strontium in laboratoryscale columns: Modelling and evaluation. Journal of Hydrology, 549, 1-11.

https://doi.org/10.1016/j.jhydrol.2017.03.053

\section{Published in:}

Journal of Hydrology

\section{Citing this paper}

Please note that where the full-text provided on Manchester Research Explorer is the Author Accepted Manuscript or Proof version this may differ from the final Published version. If citing, it is advised that you check and use the publisher's definitive version.

\section{General rights}

Copyright and moral rights for the publications made accessible in the Research Explorer are retained by the authors and/or other copyright owners and it is a condition of accessing publications that users recognise and abide by the legal requirements associated with these rights.

\section{Takedown policy}

If you believe that this document breaches copyright please refer to the University of Manchester's Takedown Procedures [http://man.ac.uk/04Y6Bo] or contact uml.scholarlycommunications@manchester.ac.uk providing relevant details, so we can investigate your claim.

\section{OPEN ACCESS}




\title{
Non-Fickian dispersive transport of strontium in laboratory-scale columns: Modelling and evaluation
}

\author{
Dongxu Liu ${ }^{1,2, *}$, Andrey P Jivkov ${ }^{2, *}$, Lichun Wang ${ }^{3}$, Gaohua $\mathrm{Si}^{1}$, Jing $\mathrm{Yu}^{1}$ \\ ${ }^{1}$ Northwest Institute of Nuclear Technology, Xi'an 710024, PR China \\ ${ }^{2}$ Research Centre for Radwaste \& Decommissioning and Modelling \& Simulation Centre, Dalton \\ Nuclear Institute, The University of Manchester, Manchester M13 9PL, UK \\ ${ }^{3}$ Department of Geological Sciences, University of Texas at Austin, Austin, Texas, USA
}

\begin{abstract}
In the context of environmental remediation of contaminated sites and safety assessment of nuclear waste disposal in the near-surface zone, we investigate the leaching and non-Fickian dispersive migration with sorption of strontium (mocking strontium-90) through columns packed with sand and clay. Analysis is based on breakthrough curves (BTCs) from column experiments, which simulated rainfall infiltration and source term release scenario, rather than applying constant tracer solution at the inlet as commonly used. BTCs are re-evaluated and transport parameters are estimated by inverse modelling using two approaches: (1) equilibrium advection-dispersion equation (ADE); and (2) continuous time random walk (CTRW). Firstly, based on a method for calculating leach concentration, the inlet condition with an exponential decay input is identified. Secondly, the results show that approximately $39 \% \sim 58 \%$ of $\mathrm{Br}^{-}$and $16 \% \sim 49 \%$ of $^{22+}$ are eluted from the columns at the end of the breakthrough experiments. This suggests that trapping mechanisms, including diffusion into immobile zones and attachment of tracer on mineral surfaces, are more pronounced for $\mathrm{Sr}^{2+}$ than for Br. Thirdly, we demonstrate robustness of CTRW-based truncated power-law (TPL) model in capturing non-Fickian reactive transport with $0<\beta<2$, and Fickian transport with $\beta>2$. The non-Fickian dispersion observed experimentally is explained by variations of local flow field from preferential flow paths due to physical heterogeneities. Particularly, the additional sorption process of strontium on clay minerals contributes to the delay of the peak concentration and the tailing features, which leads to an enhanced non-Fickian transport for strontium. Finally, the ADE and CTRW approaches to environmental modelling are evaluated. It is shown that CTRW with a sorption term can describe non-Fickian dispersive transport of strontium at laboratory scale by identifying appropriate parameters, while the traditional $\mathrm{ADE}$ with a retardation factor fails to reproduce the complex non-Fickian transport of strontium with strong sorption on clay surface.
\end{abstract}

Keywords: Non-Fickian transport; Dispersion; CTRW; Preferential flow; Sorption

\section{Introduction}

Radioactive strontium (Sr-90), a by-product of the fission of uranium and plutonium, is found in waste from nuclear activities. Since atmospheric nuclear tests conducted in the 1950s and 1960s and the accident occurred at the Chernobyl nuclear power plant, it is well known that, like many other radioactive species, large amounts of strontium- 90 were produced and dispersed worldwide in fallout into the environment (EPA, 2015). Strontium-90 is considered as one of the hazardous constituents of nuclear wastes, due to its environmental mobility, radiation exposure and biologic toxicity. Specifically, strontium-90 has chemical properties similar to calcium and therefore it tends to concentrate in the bones and teeth, and can form many chemical compounds, including halides,

\footnotetext{
* Corresponding Author. E-mail: dongxu liu nint@sina.cn; Andrey.Jivkov@manchester.ac.uk Phone: +44(0)1613063765
} 
oxides, and sulphide. This increases the risk of bone cancer, cancer of the soft tissue near the bone, and leukaemia from internal exposure (EPA, 2015). Environmental behaviour of radioactive strontium has therefore attracted increasing attention over the last three decades, focused on understanding migration of Sr-90 and strontium isotopes through field in situ tests, laboratory experiment analysis, natural analogue studies and computer modelling. However, accurate assessment and prediction of Sr-90 transport in complex environments remains unresolved, with limited number of investigations devoted to leaching, non-Fickian migration and sorption processes (Bencala, 1984; Bruno et al., 2002; Bugai et al., 2012; Eagling et al., 2013; Hull and Schafer, 2008; Kosakowski and Smith, 2005; Maderich et al., 2014; Miller et al., 1994; Qian et al., 2009; Rumynin, 2011; Volkova, 2009; Yin et al., 2014). Combining laboratory dynamic column experiments and theoretical modelling would contribute significantly to fundamental understanding and physically realistic description of strontium transport in porous media.

Understanding species (contaminants, tracers, solutes, nuclides or particles) fate and transport in the subsurface environment is of central importance in the frame of remediation schemes for contaminated sites and nuclear waste disposal (Bijeljic et al., 2004; Levchuk et al., 2012). The problem is challenging due to perennial complexities and strong heterogeneities in geological mass. From systematic viewpoint, a persistent issue is how to explore appropriate predictive models in the absence of sufficient information on heterogeneous hydraulic-physical-chemical properties. Traditionally, migration of species in geological systems, from the pore scale upwards, is described by Fickian-based advection-dispersion equation (ADE) models, which adopt deterministic and/or stochastic approaches (Bear and Cheng, 2010; Berkowitz et al., 2001; Rumynin, 2011). However, the ADE-based approaches often fail to capture and predict non-Fickian (or anomalous) transport of species in the subsurface. This is commonly observed both in the laboratory and in the field (Becker and Shapiro, 2000; Berkowitz et al., 2001; Bijeljic et al., 2013; Haggerty et al., 2001; Kang et al., 2015; Le Borgne and Gouze, 2008; Neuman and Tartakovsky, 2009), and now recognized as ubiquitous in the transport in fractured porous media.

Non-Fickian transport behaviour presents different forms and traits, commonly referred to as scale-dependent dispersion, but manifests through "unusual" early breakthrough times and long late time tails in measured breakthrough curves (BTCs), which deviate from the Gaussian distributions of species concentration (Bakshevskaia and Pozdniakov, 2015; Berkowitz et al., 2006; Edery et al., 2014). The continuous time random walk (CTRW) has been shown to be a successful framework to quantify non-Fickian transport with capacity to capture the transport transition from non-Fickian to Fickian (Berkowitz et al., 2006; Hansen and Berkowitz, 2015). The CTRW approach describes the random movement of solute particles in an Eulerian-Lagrangian framework (Neuman and Tartakovsky, 2009). The use of CTRW for species transport in porous and fractured media across different scales have been presented elsewhere (Berkowitz et al., 2006; Berkowitz and Scher, 2009; Blunt et al., 2013; Cortis and Berkowitz, 2004; Edery et al., 2013; Kang et al., 2014; Le Borgne et al., 2008; Rhodes et al., 2008; Wang and Cardenas, 2014).

In the CTRW framework, the conservative tracer (e.g. bromide) parameters, including mean fluid velocity and dispersion coefficient, are inappropriate to describe transport of reactive or sorbing tracers (e.g. atrazine, TBNPA) through laboratory columns (Li and Ren, 2009; Rubin et al., 2012). Although models for describing non-Fickian transport, including mobile-immobile, fractional derivative, and multi-rate mass transport, have been applied to simulate certain pollutants transport process (e.g. pesticides), few studies focused on the application of those models to the fate of reactive nuclides (e.g. strontium) (Dentz et al., 2011; Kapetas et al., 2014; Neuman and Tartakovsky, 2009). With respect to reactive transport it should be mentioned, that separation of migration and sorption into independent sets of measured/fitted parameters is not justifiable, although such separation has been used in the traditional ADE formulation (Rubin et al., 2012). This makes the prediction of reactive nuclide transport with pervasive non-Fickian phenomenon even more challenging. Thus, for a special reactive species, strontium in this study, there is a continuing need to 
advance the understanding and prediction of transport phenomena involving natural tracer release, non-Fickian migration and sorption.

The objectives of this paper are: (1) to re-analyse BTCs from column experiments simulating rainfall infiltration scenarios and transport behaviour of strontium (Gaohua et al., 2013); (2) to apply the ADE and CTRW models to characterize coupled effects of release, transport and sorption processes; (3) to identify transport parameters by inverse modelling against experimental data; (4) to evaluate the applicability of ADE and CTRW models in capturing reactive species transport with pervasive non-Fickian transport. This study advances the understanding and prediction of radionuclide transport through heterogeneous systems, which is critical for environmental remediation of contaminated sites and the safety assessment of nuclear waste subsurface disposal.

\section{Methodology}

\subsection{Column experiments}

Safety assessment of near-surface zone and planning remedial options for contaminated sites require detailed studies of release and transport processes. Of particular interest for near-surface nuclear waste storage is the radioactive fallout dispersed to surface in a rainfall scenario. The column experiments described here served this purpose. These were mocking rainfall infiltration with a contaminant (source) located at the inlet and slowly released to the environment, rather than the traditional supply of a tracer concentration through columns over time. Firstly, examining the tracer concentrations leaching from the source layer is essential for identifying and then modelling the inlet condition. Secondly, measuring the tracer mass eluted from the columns is necessary for the quantitative analysis of mass transport and models validation.

Column experiments, with setup shown in Fig. 1, were performed to investigate $\mathrm{Sr}^{2+}$ transport with varying sprinkling volumetric flow rates, and different packed materials, including sand and clay. $\mathrm{Br}^{-}$was used as a conservative tracer for water flow. Full details of the experimental setup and primary results are described by Gaohua et al.(2013); only information relevant to this work is given below.

The tracer experiments were conducted in a set of cylindrical organic glass columns $(3 \mathrm{~cm}$ diameter and $20 \mathrm{~cm}$ length) packed with sand and/or clay. Eight different columns were prepared: columns a, b, c, d were 18-cm long packed by medium sand of $0.25 \sim 0.50 \mathrm{~mm}$ diameter; columns e, $\mathrm{f}$, $\mathrm{g}$, $\mathrm{h}$ were packed by a mixture of clay and the same sand with $25 \%, 50 \%, 75 \%$ and $100 \%$ clay content, respectively. The packing materials were taken from the sediments in northwest China. Neat tracer sources were prepared by the following the steps: (1) Sr stock solution was made by dissolving $100 \mathrm{~g} \mathrm{SrCl}_{2} \cdot 6 \mathrm{H}_{2} \mathrm{O}$ into $250 \mathrm{~mL}$ distilled water; $\mathrm{Br}$ stock solution was made by dissolving $66 \mathrm{~g} \mathrm{NaBr}$ in $1000 \mathrm{~mL}$ distilled water; and (2) $250 \mathrm{~g}$ pure quartz sand was placed in $1000 \mathrm{~mL}$ beaker, to which $105 \mathrm{~mL} \mathrm{Sr}$ solution and $50 \mathrm{~mL} \mathrm{Br}$ solution were added, respectively; Beaker was stirred well and dried until moisture content reduced to $3 \%$.

Columns a-d were sprinkled with distilled water under different volumetric flow rates: $100 \mathrm{~mL} / \mathrm{d}$, $50 \mathrm{~mL} / \mathrm{d}, 20 \mathrm{~mL} / \mathrm{d}$ and $2 \mathrm{~mL} / \mathrm{d}$, respectively. We note that although a-d were packed with sand with the same particle size distribution, the packing structure might have varied due to preparation uncertainty. Columns e-h were sprinkled with the same flow rate $2 \mathrm{~mL} / \mathrm{d}$ of distilled water. To prevent water and soil loss, a polyester fibre membrane with an aperture $0.45 \mu \mathrm{m}$ was placed at the bottom of column. Sprinkling volumetric flow rate was controlled by injection pump and peristaltic pump as a water supply device.

During experiments, columns were saturated slowly by leaching with distilled water until steadystate fluid velocity was achieved. The first inlet section was occupied by a $0.8-\mathrm{cm}$ thick tracer source layer (Br $135.5 \mathrm{mg}$ and $\mathrm{Sr} 718 \mathrm{mg}$, approximately), which was overlain by a $0.5 \mathrm{~cm}$ thick sand. At the column outlet, effluent water was collected automatically over experimental duration. The concentration of $\mathrm{Br}^{-}$and $\mathrm{Sr}^{2+}$ was determined by ion chromatography and inductively coupled 
plasma-atomic emission spectrometry (ICP-AES), respectively. Thus, the breakthrough curves (temporal profile of concentration) of $\mathrm{Br}^{-}$and $\mathrm{Sr}^{2+}$ were obtained.

\subsection{Transport model with Advection-Dispersion Equation (ADE)}

Assuming steady-state flow and uniform water content in homogeneous porous media, the classical ADE for one-dimensional transport of species, subject to equilibrium linear isotherm adsorption and radioactive decay, can be written as (Šimůnek et al., 1999; Toride et al., 1995)

$$
R \frac{\partial C}{\partial t}=D \frac{\partial^{2} C}{\partial x^{2}}-v \frac{\partial C}{\partial x}-\lambda C
$$

where $C$ is species concentration in the liquid $\left(\mathrm{ML}^{-3}\right), R$ is dimensionless retardation factor, $D$ is dispersion coefficient $\left(\mathrm{L}^{2} \mathrm{~T}^{-1}\right), \quad v$ is average pore water velocity $\left(\mathrm{LT}^{-1}\right), \lambda$ is the first-order decay coefficient $\left(\mathrm{T}^{-1}\right), t$ is time $(\mathrm{T})$, and $x$ is transport distance $(\mathrm{L}) . R$ depends on the relative transport velocity, $v_{s}$, such that $R=v / v_{s}$, and reflects the strength of sorption, $R=1$ when no sorption occurs. $D$ can be represented by $D=v \alpha+\tau D_{w}$ (Bear, 1972), where $D_{\mathrm{w}}$ is molecular diffusion coefficient in free water $\left(\mathrm{L}^{2} \mathrm{~T}^{-1}\right), \alpha$ is dispersivity $(\mathrm{L}), \tau$ is a dimensionless tortuosity factor. For stable species $\lambda=0$.

For an initially species-free and semi-finite column, where tracer is placed in a fixed layer at the inlet of column and is eluted with free water, the leach concentration of effluent from the bottom of the source layer will decrease over time as the tracer mass in the source layer reduces due to mass loss through leaching. The leach concentration can be estimated via an exponential function using the leaching rate coefficient (Chen et al., 2007)

$$
C(t, 0)=\frac{\lambda_{L} m_{0}}{Q} \mathrm{e}^{-\left(\lambda_{L}+\lambda\right) t}
$$

where $C(t, 0)$ is the leach concentration $\left(\mathrm{ML}^{-3}\right), m_{0}$ is the total mass of tracer $(\mathrm{M}), Q$ is the sprinkling volumetric flow rate $\left(\mathrm{L}^{3} \mathrm{~T}^{-1}\right), \lambda_{L}$ is a leaching rate coefficient $\left(\mathrm{T}^{-1}\right)$. A first-order decay rate of the source term, similar to radioactive decay, was given by Baes and Sharp(1983) and Chen et al.(2007)

$$
\lambda_{L}=\frac{Q}{\varepsilon R V_{t}}=\frac{I}{\varepsilon R d}
$$

where $\varepsilon, V_{t}, d$ are effective porosity $\left(\mathrm{L}^{3} \mathrm{~L}^{-3}\right)$, volume $\left(\mathrm{L}^{3}\right)$, and depth $(\mathrm{L})$ of the tracer source layer, respectively, $I$ is infiltration rate of water $\left(\mathrm{LT}^{-1}\right), R$ is the retardation factor of tracer in the source layer (-).

The model of leach concentration represented by Eqs.(2) (3) is a relatively simple approximation of the complex transport mechanism involving physical and chemical processes. This model assumes that equilibrium adsorption is achieved instantaneously between the passing water and the source layer when the percolating water comes into contact with the tracer source layer. The equilibrium is generally described by a linear adsorption relationship using a distribution coefficient $\left(K_{d}\right)$ estimated from batch experiments (Baes and Sharp, 1983; Chen et al., 2007). $R$ can be thus calculated based on $K_{d}$, such that $R=1+\rho_{b} \cdot K_{d} / \varepsilon$, where $\rho_{b}$ is bulk density, and $\varepsilon$ is effective porosity. Moreover, combining with the inverse modelling, $R$ can be obtained directly from column experiments (Rod et al., 2010).

The simulated BTCs can be solved with given boundary conditions. Here, Dirichlet boundary with prescribed concentration, which is determined by the leach concentration curve as described by Eq.(2), is set for the inlet boundary of the column. Further, Neuman boundary condition with no dispersive flux is set for the outlet boundary.

\subsection{Transport model with Continuous Time Random Walk (CTRW)}

The concept and the formulation of the CTRW method can be found in various references (Berkowitz et al., 2006; Cortis et al., 2004; Dentz et al., 2004; Edery et al., 2015; Edery et al., 2013; Neuman and Tartakovsky, 2009; Scher and Lax, 1973). Here, we sketch the key features of CTRW for the purpose of this study. 
In the CTRW framework, transport processes are conceptualized as a series of particle transitions by statistically characterizing tracer motion. The main feature of CTRW lies in quantifying temporal transition probability of particle transport induced by spatial heterogeneities via a memory function (Berkowitz et al., 2006).

The one-dimensional (1D) CTRW for macroscopic transport through a uniform domain is given in Laplace space by Eqs. (4)-(7) (Cortis and Berkowitz, 2005; Edery et al., 2014)

$$
u \tilde{c}(x, u)-c_{0}(x)=-\tilde{M}(u)\left[v_{\psi} \frac{\partial}{\partial x} \tilde{c}(x, u)-D_{\psi} \frac{\partial}{\partial x^{2}} \tilde{c}(x, u)\right]
$$

$$
\begin{gathered}
\tilde{M}(u) \equiv \overline{t u} \frac{\tilde{\psi}(u)}{1-\tilde{\psi}(u)} \\
v_{\psi}=\frac{1}{n \bar{t}} \sum_{s} p(\boldsymbol{s}) \boldsymbol{s} \\
D_{\psi}=\frac{1}{n \bar{t}} \sum_{s} \frac{1}{2} p(\boldsymbol{s}) \boldsymbol{s} \boldsymbol{s}
\end{gathered}
$$

where $u$ is the Laplace variable and the tilde represents the Laplace transformed variable; $c_{0}$ is the initial concentration and $\tilde{c}(x, u)$ is the Laplace-transformed normalized concentration; $\tilde{M}(u)$ is a memory function; $\psi(t)$ is a probability density function (PDF), defined as the probability rate for a transition time $t$ between transport sites and $\tilde{\psi}(u)$ is the Laplace-transformed form of $\psi(t) ; v_{\psi}$ and $D_{\psi}$ are the tracer transport velocity and dispersion coefficient, respectively; $\bar{t}$ is a characteristic time; $n$ is porosity; $\boldsymbol{s}$ is the position vector and $p(\mathbf{s})$ is the probability distribution of the length of transitions. Note that both $v_{\psi}$ and $D_{\psi}$ depend on $\psi(t)$, which is fundamentally different from the average pore water velocity $(v)$ and dispersion coefficient $(D)$ in the classical ADE (Berkowitz et al., 2006).

The PDF, $\psi(t)$, is the core of the CTRW formulation as it depicts the nature of solute plume migration patterns (Berkowitz et al., 2001). Depending on the choice of $\psi(t)$, the CTRW can capture a broad range of transport regimes, including the transition from non-Fickian to Fickian transport. There are several compelling forms of $\psi(t)$ that have been proposed in the literature (Berkowitz et al., 2006; Cortis and Berkowitz, 2005; Cortis et al., 2004; Margolin et al., 2003). Here, we present one specific form for conservative tracer transport, which is widely used to interpret transport phenomena at different scales: the truncated power law (TPL) model

$$
\tilde{\psi}(u) \equiv\left(1+\tau_{2} u t_{1}\right)^{\beta} \exp \left(t_{1} u\right) \Gamma\left(-\beta, \tau_{2}^{-1}+t_{1} u\right) / \Gamma\left(-\beta, \tau_{2}^{-1}\right), 0<\beta<2
$$

where $\beta$ is a critical parameter characterizing the regimes of the dispersive transport, $t_{1}$ is a characteristic transition time for the onset of the power law region, $t_{2}$ is a "cut-off" time, $\tau_{2}=t_{2} / t_{1}$. The memory function is determined by setting $\bar{t}=t_{1}$.

The TPL model allows for a systematic investigation of non-Fickian transport observed for transition times $t_{1}<t<t_{2}$, and for analysing the transition from non-Fickian to Fickian transport for $t>t_{2}$ (Dentz et al., 2004). The $t_{1}$ sets the lower limit from which the power law behaviour begins, so that the time range of interest is $t>t_{1}$. The time $t_{2}$ governs the crossover from power law to a decreasing exponential function (Edery et al., 2014; Gao et al., 2009). Overall, the $\psi(t)$ exhibits a wide change in behaviour as a function of $\beta$ that largely determines $\psi(t)$ (Edery et al., 2014). That is, $\beta$ controls the particle transport and thus functionally captures the dispersion regimes (i.e. Fickian or non-Fickian) (Cortis et al., 2004). The relative shapes of the anomalous transport regimes are strongly dependent on $\beta$, as shown in Table 1 .

Additionally, the sorbing tracer transport with pervasive non-Fickian features can be modelled explicitly through the CTRW with a modified term given by Margolin et al.(2003) and Cortis et al. (2006). The flexibility of CTRW lies in the reconstruction of $\psi(t)$ by adding an extra term $(\Lambda)$ when sorption occurs, where $\psi(t)$ can be determined on the basis of a prior-known $\psi_{0}$ for a conservative tracer in the same flow system. In the Laplace space, this is given by 


$$
\varphi /(x, u)=\varphi / \%(x, u+\Lambda-\Lambda \%((u))
$$

where $\Lambda$ is the average "sticking" rate, $\phi((u)$ is a single "sticking time" pdf.

For small-scale physical-chemical heterogeneities, $\varphi(u)$ can be characterized by the form Cortis et al.(2006)

$$
q(u)=\frac{1}{1+u^{\mu}}
$$

where $\mu$ is a positive and smaller than 1 which yields power law tailing.

In this study, the TPL model with sorption is used to interpret the measured BTCs of $\mathrm{Sr}^{2+}$, since the TPL model has been proved to be effective in describing non-Fickian transport for numerous laboratory and field observations (Edery et al., 2014). Specifically, the Laplace transformed analytical solution to the CTRW equations is obtained for a set of given boundary conditions: Dirichlet boundary condition (prescribed concentration) with an exponential decay function of concentration is specified at the inlet of the columns according to the Eq. (2); Neumann boundary condition (prescribed flux) is specified at the outlet boundary.

\subsection{Inverse modelling with ADE and CTRW-TPL methods}

The experimental BTCs are analyzed using the equilibrium ADE and CTRW-TPL methods described above. Inverse modelling for parameters identification is implemented by fitting analytical solutions with the models to the observed BTCs. The inversion processes are conducted through the non-linear least squares inversion program, CXTFIT (Šimůnek et al., 1999; Toride et al., 1995), and the CTRW MATLAB toolbox which is available from the website http://www.weizmann.ac.il/ESER/People/Brian/CTRW.

From the measured effluent concentration $C_{f}$ at the outlet boundary, and the concentration at the onset of leaching process $C_{0}$ at the bottom of the source layer (both in $\mathrm{ML}^{-3}$ ), a normalized concentration $c$ is defined by

$$
c=\frac{C_{f}}{C_{0}},
$$

Further, the ratio of eluted tracer mass to the total tracer mass $m_{0}$, i.e. the cumulative mass fraction eluted from columns $f$ is calculated by

$$
f=\frac{Q \int_{0}^{t} C_{f} d t}{m_{0}} .
$$

The two parameters, $c$ and $f$, are used to constrain the inversion process.

The inverse modelling with the equilibrium ADE for $\mathrm{Br}^{-}$and $\mathrm{Sr}^{2+}$ is performed with CXTFIT. The inverse problem is solved by minimizing an objective function of root mean square error between observed and fitted concentrations (Šimünek et al., 1999). For Br", the retardation coefficient $R$ is set to 1 , because it is assumed that $\mathrm{Br}^{-}$have minimal sorption onto sand and clay (Srinivasan and Sarmah, 2014). The parameters identified for $\mathrm{Br}^{-}, v$ and $D$, are then used as input values for the measured $\mathrm{Sr}^{2+}$ BTCs, so that $R$ for strontium can be estimated.

Further, the inversion of 1D TPL model for $\mathrm{Sr}^{2+}$ is implemented by comparing the predicted BTCs from the CTRW to the normalized BTCs from tracer experiment. The inverse modelling requires initial guesses of seven fitted parameters, including $v_{\psi}, D_{\psi}, \beta, t_{1}, t_{2}, \Lambda$ and $\mu$. Depending on how close the initial values of the parameters are to the optimized ones, the inverse estimation of parameters can be time-consuming. Here the values of $v$ and $D$ from CXTFIT are used as initial guesses of $v_{\psi}$ and $D_{\psi}$. Considering that the TPL model is sensitive to the exponent $\beta$ while relatively insensitive to $t_{1}$ and $t_{2}$ (Cortis and Berkowitz, 2005), a broad range for $\beta$ is tested, from 0.8 to 1.8 , while $t_{1}$ and $t_{2}$ are estimated from global error minimisation. Further, $\Lambda$ and $\mu$, which influence the degree of 
retardation and the power law slope of the BTC tailing (Cortis et al., 2006; Margolin et al., 2003), are initially set to $0.01 \sim 0.3$ and $0.01 \sim 0.2$, respectively. The approach to estimate initial values and to perform adjustments using trial and error method corresponds to the successful sensitivity analysis (Cortis et al., 2006). This allowed for achieving good agreement between experimental and modelling results with the CTRW toolbox.

\section{Results and Discussion}

\subsection{Tracer leach concentration and eluted mass fraction}

Leach concentration of $\mathrm{Br}^{-}$and $\mathrm{Sr}^{2+}$ in the experiments are estimated by Eqns. (2) and (3), and presented in Fig. 2. Assuming that there is no strontium sorption to quartz in the source layer (Rod et al., 2010), $\mathrm{Sr}^{2+}$ concentration demonstrates an exponential decay over the experimental duration, similar to $\mathrm{Br}^{-}$. Because the leaching rate, $\lambda_{L}$, is proportional to the volumetric flow rate, $Q$, the leach concentration is inversely exponentially dependent on $Q$, i.e. very strongly. The higher the flow rate is, the more easily the tracer particles transfer to aqueous phase and are subjected to stronger hydrodynamic dispersion with time. This eventually leads to faster falloff of leach concentration with shorter residence time, and vice versa (Fig. 2). Moreover, as more mass of $\mathrm{Sr}^{2+}$ than $\mathrm{Br}^{-}$is located at the source layer, the $\mathrm{Sr}^{2+}$ concentration is greater than that of $\mathrm{Br}^{-}$at the onset of leaching. The release of source term is characterized as a continuous and decreasing process rather than an instantaneous process. This is due to mass loss through leaching over time. As a result, the inlet condition of the leaching release process should be treated as an exponential decay function, instead of a simple instantaneous pulse or Dirac delta function, which is commonly employed for experimental and numerical simplicity. This treatment of tracer release is closer to the natural condition in simulating rainfall infiltration, and is critical for dynamic simulation of tracer transport, e.g. in the CTRW modelling.

The cumulative eluted mass fraction for $\mathrm{Br}^{-}$is approximately $39 \% \sim 58 \%$ for the observed columns, as given in Fig. 3. The results show that around half of the mass remains in the source layers and soil columns. This indicates that a fraction of pore water is immobile due to the presence of "dead pore" in relation to fluid flow. For $\mathrm{Sr}^{2+}$, the cumulative eluted mass fraction is approximately $16 \% \sim 49 \%$ (Fig. 3), which is much less than that of $\mathrm{Br}^{-}$. This signifies that, more clay content results in larger sorption of strontium and less aqueous strontium, as seen for columns e-h. Herein, the effect of volumetric flow rate on transport is excluded in the study since the packing structures for columns ad may vary from one to another due to uncertainty in the consistency of packing. It is worth noting, however, that larger eluted mass fraction is observed for $\mathrm{Br}^{-}$and $\mathrm{Sr}^{2+}$ in column $\mathrm{d}$, which can be attributed to relatively larger heterogeneity in this packing, as discussed in the next sub-section.

\subsection{Observations of non-Fickian transport}

The typical non-Fickian features, BTCs with early breakthrough and late tailings, are observed in varying degrees in our study. Particularly pronounced is the experiment with column $\mathrm{d}$. This is illustrated by the concentration results shown in Fig. 4, which are also used to show the predictions of the two methods after parameter estimation, i.e. best fits. Although column $d$ is packed with "homogeneous" sand and sprinkled with a relatively slow flow rate, $2 \mathrm{~mL} / \mathrm{d}$, the "anomalous" transport phenomenon is remarkable. This might be due to the occurrence of preferential flow path caused by the heterogeneous packing structure. Nevertheless, non-Fickian transport of strontium is observed in some BTCs, which implies that physical-chemical heterogeneities contribute strongly via preferential flow, diffusion in/out of immobile zones and complex sorption.

The estimated parameters of the two tested models are given in Table 2. Estimated $0<\beta<1$ for columns $\mathrm{d}, \mathrm{g}$, and $\mathrm{h}$, indicates highly dispersive transport leading to longer tailing. The early breakthrough time observed for column $\mathrm{d}$ (Fig. 4d) is not expected in view of the lower flow rate compared to columns a-c. This suggests the existence of stronger preferential flow path as well as larger immobile zones. Potentially, a fraction of strontium is trapped or diffuses into low 
permeability lenses and remains there for long times. The strontium particles that firstly reach the outlet with short residence time are those that propagate through the faster moving regions along preferential flow paths. Thus, the "homogeneous" column $\mathrm{d}$ with a much smaller $\beta$, larger transport velocity and dispersion coefficient, fails to comply with the Fick transport theory and exhibits significant non-Fickian dispersion.

For columns $\mathrm{g}$ and $\mathrm{h}$ (Fig. $4 \mathrm{~g}$ and $\mathrm{h}$ ), in addition to diffusion into immobile zones, sorption is a reasonable explanation for the non-Fickian migration based on the conclusion of (Rubin et al., 2012). The reduction and delay of the peak concentration and the tailing features could be affected by the additional processes of adsorption/desorption of strontium onto/from the mineral surface. This partitioning mechanism between aqueous and mineral phases enhances the non-Fickian transport and causes the limitation of Fickian-based equilibrium ADE model.

Comparatively, columns a, e and $\mathrm{f}$ with $1<\beta<2$, present moderate non-Fickian processes at different water flow fields and particles residence time. Note that in Fig. 4a, the BTCs demonstrate early arrival and late tail, corresponding to the larger transport velocity and dispersion coefficient, which are similar to the phenomenon of column $d$ and distinct from columns e and $f$. This can be explained by the higher pore water velocities enhancing the preferential flow in the presence of immobile water (Gaber et al., 1995; Li and Ren, 2009). For columns e and f, sorption retardation process (e.g. strontium cations exchange with inorganic cations in clay), serves as an additional factor contributing weakly to the non-Fickian dispersive transport. The contribution of sorption to the non-Fickian features depends on the amount of clay as seen in Fig. 4e-h.

Unlike column a with high volumetric flow rate, Fickian transport at medium volumetric flow rate is predicted fairly well for column $\mathrm{b}$ and $\mathrm{c}$ with values of $\beta$ slightly larger than 2 (Fig. $4 \mathrm{~b}$ and $\mathrm{c}$ ). This suggests that the assumption of equilibrium mass transfer between spectrums of different mobility is adequate for moderate flow rates within relatively homogeneous structures. The results also supports our assumption that little sorption/desorption occurs for sand-packed columns.

Our results strengthen the understanding that both physical and chemical heterogeneities may influence strongly species transport (Mohamed et al., 2010). For laboratory scale disturbed and undisturbed soil columns, the role of physical non-equilibrium transport stemming from physical heterogeneities, i.e. different pore scale structures, is critical for inducing non-Fickian phenomena: macro-pores commonly control the preferential flow process, while some pore spaces serve as tracer sinks at early times, and then release tracer slowly. This drives non-Fickian transport with late tailing feature (Dousset et al., 2007; Swanson et al., 2015). It is known that preferential flow and transport may exist in unconsolidated sandy soils. This applies to soils with available structural voids as a result of textural shift horizontally, funneling effect, or fluid instabilities due to viscosity or density differences between the invading and resident water (Kamra et al., 2001). Further, chemical heterogeneities across various scales, which control chemical equilibrium (instantaneous sorption) or non-equilibrium (kinetic sorption) transport processes, apparently contribute the non-Fickian mechanisms.

\subsection{Physical heterogeneity effects on non-Fickian transport}

Due to minimal sorption in the sand-packed columns, column experiments a-d allow for evaluating transport behaviour quantitatively due to physical aspects alone - advection and dispersion. The main parameters influencing advective-dispersive transport are the fluid velocity and dispersion coefficient. Although a correlation between the tracer transport velocities and the volumetric flow rates is found by the ADE model (Table 2), the resultant fitting parameters do not follow the expected Fickian trend, because the ADE is insufficient to capture the highly non-Fickian transport in column $\mathrm{d}$. In contrast, transport velocity and dispersion coefficient for column $\mathrm{d}$ estimated by the CTRW-TPL are greater than those for columns $b$ and $c$. This implies that columns $b$ and $\mathrm{c}$ are likely forming more homogeneous packed structures, while column $\mathrm{d}$ is likely to have a 
relatively heterogeneous one. That is, the presence of macro-pores in column d may lead to overall faster transport and high dynamic dispersion.

Similar to fracture-matrix systems, where fracture leads to fast breakthrough and matrix contributes to the long tailing of BTCs (Kosakowski and Smith, 2005), the eluted mass fraction and inverse modelling for the BTCs indicate that the presence of interconnected open channels, e.g. macro-pores or preferential flow paths, in conjunction with low velocity regions, e.g. in dead-end channels or immobile zones, is responsible for the non-Fickian dispersion transport in heterogeneous columns. Specifically, local flow velocity variations, i.e. low-velocity fluid near channel walls and higher-velocity fluid near the centre of the channel, as well as the matrix diffusion, result in a skewed breakthrough curve with an earlier peak and longer tail.

Pore-scale heterogeneity and variations of fluids velocity in the pore space are known to yield enhanced dispersive transport, associated with the Peclet number $(\mathrm{Pe})$ (Dentz et al., 2011). Depending on the selected length scale, $P e$ is presented in different forms (Bear and Cheng, 2010). At the macroscopic level, $P e=v L / D=L / \alpha$, where $L$ is the column length, represents the ratio between the advective and dispersive transport terms. It increases when the advection dominates over dispersion (Šimůnek et al., 2008). For $P e<<1$, the advective mass flux may be neglected as much smaller than the dispersive one, and vice versa (Bear and Cheng, 2010). In this study, based on the fitted parameters (Table 2) and the column length, $L=18 \mathrm{~cm}, P e$ is calculated for the experimental columns with results presented in Table 3. The results, $1<P e<200$, show that both dispersion and advection are operating, e.g. there is a transition region to a power-law regime (Dentz et al., 2011). In this case, the CTRW-based TPL model is valid for transport regimes. The possible effects of $P e$ on dispersive transport are not analysed here, but are subject of further investigations.

\subsection{Chemical heterogeneity effects on non-Fickian transport}

The column experiments e-h are designed to evaluate the effect of sorption on strontium transport. Expectedly, the tracer transport velocities decrease with increased clay content (Table 2), as strontium transport is significantly retarded. The emergent behaviour is non-Fickian dispersive transport, i.e. reduction and delay of the peak concentration and elongation of tailing. The larger the clay content, the greater the retardation and the reduction of $\mathrm{Sr}^{2+}$ (Fig. 4e-h, Table 2). Clay content was positively correlated to the degree of non-Fickian transport, which was indicated by low beta values (see Tables 1 and 2). Those phenomena are fundamentally due to the sorption/desorption of strontium on clay surface.

Sorption coefficients, represented in the form of $R$ or $K_{d}$, are commonly used to approximate the extent of species sorption on various minerals, where typical sorption reactions are surface complexation and ion exchange (Altmann et al., 2001). As sorption is commonly a complex function of chemical properties, e.g. $\mathrm{pH}$, ionic strength, mineral surface area, etc., the integral (constant) coefficient $R$ or $K_{d}$ used in ADE model often fail to capture such complexities. This is demonstrated by our results that the equilibrium ADE is insufficient to fully capture measured BTCs. Moreover, for spatially heterogeneous retardation properties, which can give rise to distinct non-Fickian transport behaviours, the sorption coefficient $R$ may not exist at relevant time and length scales (Dentz and Castro, 2009). Consequently, the prediction of increasing $R$ with increasing clay content by the ADE model (Table 2) is not sufficient to describe $\mathrm{Sr}^{2+}$ transport in clayed media using the equilibrium sorption model (Fig. 4e-h), because $R$ reflects the simplified kinetic sorption process to some degree but fails to capture the chemical heterogeneities, e.g. spatially varying sorption properties and specific reactive surface areas (Dentz et al., 2011).

In contrast, the CTRW framework with a sticking rate term is found capable of representing $\mathrm{Sr}^{2+}$ transport (Fig. 4e-h), where slow and fast species transport is manipulated by the random spatial and time increment as a stochastic process, and heterogeneity is mapped on the joint distribution of transition length and times (Dentz et al., 2011). As expected, the average "sticking" rate $\Lambda$ increases with increasing clay content (Table 2). This demonstrates that $\Lambda$ represents physically the degree of 
sorption of $\mathrm{Sr}^{2+}$ on clay minerals, and shows that our CTRW-TPL model is robust in capturing nonFickian transport when sorption is operating (Fig. 4e-h). Overall, the CTRW model is of the greater flexibility to describe the local scale transport with spatially varying equilibrium mass transfer and sorption-desorption reactions (Bromly and Hinz, 2004; Dentz and Castro, 2009), and thus can capture observed non-Fickian transport features, e.g. highly non-Gaussian BTCs.

\subsection{Performance of ADE and CTRW methods in environmental modelling}

Environmental modelling uses mathematics and computers to simulate physical and chemical phenomena across different environmental scales (Holzbecher, 2012). For nuclide transport related to contaminated sites remediation and safety assessments of a nuclear waste disposal, a number of macroscopic models based on ADE have been proposed, e.g. dual-porosity model, dual permeability model, two-phase model (Kapetas et al., 2014; Simůnek and van Genuchten, 2008). The CTRW framework has mainly been applied for reactive transport, e.g. chemical sorption on minerals (Deng et al., 2013; Dentz et al., 2011; Edery et al., 2015).

In this work, inverse modelling by CXTFIT and CTRW toolbox have been used to obtain the relevant model parameters (Table 2). Overall, the CTRW performs better than the ADE, specifically in capturing measured BTCs, even though there should be little difference between them for column d, due to negligible sorption (Fig. 4). The equilibrium ADE modelling leads to broad range of parameters in capturing asymmetric BTCs by CXTFIT (Table 2), as the simple average deterministic values may be insufficient to capture pervasive non-Fickian transport and estimate the randomness of tracer transference. The CTRW-TPL model appears to be the preferred method to describe this nonFickian transport. Further, the CTRW-TPL is found insensitive to the magnitude of the Peclet number and offers an efficient alternative for modelling species transport in convection-dominant flows (COMSOL, 2013). It is noted that some BTCs fitted with the ADE through CXTFIT are similar to ones with TPL through CTRW toolbox (Fig. 4). This can be explained by the relative flexibility of CXTFIT to fit certain BTCs. However, this flexibility appears to have limited validity, excluding strongly preferential flow conditions (Kamra et al., 2001).

Both ADE and CTRW methods have been widely credited as effective tools to simulate species transport in heterogeneous conditions, utilising Lagrangian (particle tracing) and Eulerian (integraldifferential equation) frameworks (Bakshevskaia and Pozdniakov, 2015; Hansen and Berkowitz, 2015; Rumynin, 2011). In fact, CTRW offers a general formalism which encompasses the ADE models, and thus has comprehensive ability in treating non-Fickian dispersion transport (Berkowitz et al., 2006; Gao et al., 2009). Moreover, the probabilistic base of CTRW provides a more realistic mathematical framework to model the advection-dispersion in porous media than the deterministic base of ADE (Kulasiri, 2013). However, there are unresolved issues with respect to the nonuniqueness of fitted parameters and inverse solution because of internal correlations between CTRW parameters. This results in the existence of many local minima in the parameter estimation method (Kulasiri, 2013). To surmount the problem, apart from collecting supplementary information which is independent of the fitted parameter and reflects the actual migration processes, it is suggested to integrate the ADE and CTRW approaches to find optimal parameters to improve inversion problem. For example, in this work we set admissible limits to the effective transport velocity and dispersion coefficient found by ADE model as constraints to the CTRW. Then, in the CTRW framework, $\beta$ can be estimated through analysis of the velocity field (Gao et al., 2009). Finally, the optimization scheme allows for calculating reliable parameter values, which provide robust model prediction.

From a practical perspective, however, a disadvantage of CTRW is the lack of well-established public domain or commercial package; only CTRW MATLAB toolbox is available as mentioned in the section 2 . In contrast, there are widely available user-friendly software packages for ADE, such as CTXFIT, HYDRUS (Šimůnek et al., 2008), MODFLOW (Chiang and Kinzelbach, 2005), upon which customized models can be built without sophisticated programming and coding. 


\section{Conclusions}

Understanding release and transport of radioactive strontium is important in the context of remedial options for contaminated sites and safety assessment of disposal in near-surface zone. Few investigations have focused on strontium non-Fickian transport with coupled advection, dispersion and sorption processes, through an integrated approach of laboratory dynamic column experiments and computer modelling. By re-analyzing BTCs from realistic column experiments of strontium transport behaviour, comprehensive analysis for release and non-Fickian dispersive migration of strontium was carried out using the ADE-based CTXFIT and TPL-based CTRW toolbox. The results allow for the following conclusions:

(1) The proposed, relatively simple, method for calculating leach concentration from the source layer allows us to understand a continuous and decreasing tracer release process (Fig. 2). This satisfies an approximation of exponential decay function for the inlet condition to constrain the ADE and CTRW framework.

(2) The CTRW-TPL model can capture non-Fickian transport with $0<\beta<2$ and Fickian transport with $\beta>2$ (Table 2, Fig. 4). The non-Fickian dispersive transport could be due to variation of flow fields and sorption retardation processes, which are potentially caused by heterogeneities in the physical and chemical properties and processes.

(3) The CTRW-TPL performs better than the ADE model in terms of capturing pervasive nonFickian transport, however, both ADE and CTRW models have positive and negative aspects for practical use: integration of $\mathrm{ADE}$ and CTRW is suggested to obtain reliable parameters and provide robust model prediction for both conservative and reactive transport (see sub-sections 2.4 and 3.5).

\section{Acknowledgments}

This research reported here was partially funded by the Project of Intelligence Importation from Northwest Institute of Nuclear Technology (NINT) of China. We would like to express our sincere appreciations to Prof. Brian Berkowitz for many useful discussions and kindly help in CTRW application.

\section{References}

Altmann, S., Bruno, J., Tweed, C., 2001. Using thermodynamic sorption models for guiding radioelement distribution coefficient (Kd) investigations for performance assessment: A status report. Nuclear Energy Agency, Paris, France.

Baes, C., Sharp, R., 1983. A proposal for estimation of soil leaching and leaching constants for use in assessment models. Journal of Environmental Quality, 12(1): 17-28.

Bakshevskaia, V.A., Pozdniakov, S.P., 2015. Simulation of Hydraulic Heterogeneity and Upscaling Permeability and Dispersivity in Sandy-Clay Formations. Mathematical Geosciences: 1-20.

Bear, J., 1972. Dynamics of fluids in porous media. Courier Corporation.

Bear, J., Cheng, A.-D., 2010. Modeling groundwater flow and contaminant transport, 23. Springer Science \& Business Media.

Becker, M.W., Shapiro, A.M., 2000. Tracer transport in fractured crystalline rock: Evidence of nondiffusive breakthrough tailing. Water Resources Research, 36(7): 1677-1686.

Bencala, K.E., 1984. Interactions of solutes and streambed sediment: 2. A dynamic analysis of coupled hydrologic and chemical processes that determine solute transport. Water Resources Research, 20(12): 1804-1814.

Berkowitz, B., Cortis, A., Dentz, M., Scher, H., 2006. Modeling non - Fickian transport in geological formations as a continuous time random walk. Reviews of Geophysics, 44(2).

Berkowitz, B., Kosakowski, G., Margolin, G., Scher, H., 2001. Application of continuous time random walk theory to tracer test measurements in fractured and heterogeneous porous media. Groundwater, 39(4): 593-604. 
Berkowitz, B., Scher, H., 2009. Exploring the nature of non-Fickian transport in laboratory experiments. Advances in Water Resources, 32(5): 750-755.

Bijeljic, B., Muggeridge, A.H., Blunt, M.J., 2004. Pore - scale modeling of longitudinal dispersion. Water Resources Research, 40(11).

Bijeljic, B., Raeini, A., Mostaghimi, P., Blunt, M.J., 2013. Predictions of non-Fickian solute transport in different classes of porous media using direct simulation on pore-scale images. Physical Review $E$, 87(1): 013011.

Blunt, M.J. et al., 2013. Pore-scale imaging and modelling. Advances in Water Resources, 51: 197-216.

Bromly, M., Hinz, C., 2004. Non - Fickian transport in homogeneous unsaturated repacked sand. Water Resources Research, 40(7).

Bruno, J., Duro, L., Grivé, M., 2002. The applicability and limitations of thermodynamic geochemical models to simulate trace element behaviour in natural waters. Lessons learned from natural analogue studies. Chemical geology, 190(1): 371-393.

Bugai, D. et al., 2012. Radionuclide migration at experimental polygon at Red Forest waste site in Chernobyl zone. Part 2: Hydrogeological characterization and groundwater transport modeling. Applied Geochemistry, 27(7): 1359-1374.

Chen, Q., Kowe, R., Mobbs, S., Jones, K., 2007. Radiological Assessment of Disposal of Large Quantitites of Very Low Level Waste in Landfill Sites. Health Protection Agency, Radiation Protection Division.

Chiang, W.-H., Kinzelbach, W., 2005. 3D-groundwater modelling with PMWIN, a simulation system for modelling groundwater flow and transport. Springer, New York.

COMSOL, I., 2013. Particle Tracing Module Model Library Manual. http://hpc.mtech.edu/comsol/pdf/Particle_Tracing_Module/ParticleTracingModelLibraryManual.pdf

Cortis, A., Berkowitz, B., 2004. Anomalous transport in "classical" soil and sand columns. Soil Science Society of America Journal, 68(5): 1539-1548.

Cortis, A., Berkowitz, B., 2005. Computing "anomalous" contaminant transport in porous media: The CTRW MATLAB toolbox. Groundwater, 43(6): 947-950.

Cortis, A., Gallo, C., Scher, H., Berkowitz, B., 2004. Numerical simulation of non - Fickian transport in geological formations with multiple - scale heterogeneities. Water Resources Research, 40(4).

Cortis, A. et al., 2006. Transport of Cryptosporidium parvum in porous media: Long - term elution experiments and continuous time random walk filtration modeling. Water resources research, 42(12).

Deng, H. et al., 2013. Upscaling retardation factor in hierarchical porous media with multimodal reactive mineral facies. Chemosphere, 91(3): 248-257.

Dentz, M., Castro, A., 2009. Effective transport dynamics in porous media with heterogeneous retardation properties. Geophysical Research Letters, 36(3).

Dentz, M., Cortis, A., Scher, H., Berkowitz, B., 2004. Time behavior of solute transport in heterogeneous media: transition from anomalous to normal transport. Advances in Water Resources, 27(2): 155173.

Dentz, M., Le Borgne, T., Englert, A., Bijeljic, B., 2011. Mixing, spreading and reaction in heterogeneous media: A brief review. Journal of contaminant hydrology, 120: 1-17.

Dousset, S., Thevenot, M., Pot, V., Šimunek, J., Andreux, F., 2007. Evaluating equilibrium and non-equilibrium transport of bromide and isoproturon in disturbed and undisturbed soil columns. Journal of contaminant hydrology, 94(3): 261-276.

Eagling, J., Worsfold, P.J., Blake, W.H., Keith-Roach, M.J., 2013. Fate of $90 \mathrm{Sr}$ and U (VI) in Dounreay sediments following saline inundation and erosion. Chemosphere, 92(8): 911-917.

Edery, Y., Dror, I., Scher, H., Berkowitz, B., 2015. Anomalous reactive transport in porous media: Experiments and modeling. Physical Review E, 91(5): 052130.

Edery, Y., Guadagnini, A., Scher, H., Berkowitz, B., 2013. Reactive transport in disordered media: Role of fluctuations in interpretation of laboratory experiments. Advances in Water Resources, 51: 86-103.

Edery, Y., Guadagnini, A., Scher, H., Berkowitz, B., 2014. Origins of anomalous transport in heterogeneous media: Structural and dynamic controls. Water Resources Research, 50(2): 1490-1505. 
EPA, 2015. Radiation Protection Reference Information-Strontium. http://www.epa.gov/radiation/radionuclides/strontium.html updated on June 29, 2015.

Gaber, H., Inskeep, W., Wraith, J., Comfort, S., 1995. Nonequilibrium transport of atrazine through large intact soil cores. Soil Science Society of America Journal, 59(1): 60-67.

Gao, G., Zhan, H., Feng, S., Huang, G., Mao, X., 2009. Comparison of alternative models for simulating anomalous solute transport in a large heterogeneous soil column. Journal of hydrology, 377(3): 391404.

Gaohua, S., Xu, Y.J.L.D.Z., Zhiqian, H., 2013. The column experiment and numerical simulation for nuclide migration. Environmental Chemistry, 3: 024.

Haggerty, R., Fleming, S.W., Meigs, L.C., McKenna, S.A., 2001. Tracer tests in a fractured dolomite: 2. Analysis of mass transfer in single-well injection-withdrawal tests.

Hansen, S.K., Berkowitz, B., 2015. Integrodifferential formulations of the continuous-time random walk for solute transport subject to bimolecular $A+B \rightarrow 0$ reactions: From micro-to mesoscopic. Physical Review E, 91(3): 032113.

Holzbecher, E., 2012. Environmental modeling: using MATLAB. Springer Science \& Business Media.

Hull, L.C., Schafer, A.L., 2008. Accelerated transport of $90 \mathrm{Sr}$ following a release of high ionic strength solution in vadose zone sediments. Journal of contaminant hydrology, 97(3): 135-157.

Kamra, S., Lennartz, B., Van Genuchten, M.T., Widmoser, P., 2001. Evaluating non-equilibrium solute transport in small soil columns. Journal of Contaminant Hydrology, 48(3): 189-212.

Kang, P.K. et al., 2014. Pore - scale intermittent velocity structure underpinning anomalous transport through 3 - D porous media. Geophysical Research Letters, 41(17): 6184-6190.

Kang, P.K., Le Borgne, T., Dentz, M., Bour, O., Juanes, R., 2015. Impact of velocity correlation and distribution on transport in fractured media: Field evidence and theoretical model. Water Resources Research, 51(2): 940-959.

Kapetas, L., Dror, I., Berkowitz, B., 2014. Evidence of preferential path formation and path memory effect during successive infiltration and drainage cycles in uniform sand columns. Journal of contaminant hydrology, 165: 1-10.

Kosakowski, G., Smith, P., 2005. Modelling the transport of solutes and colloids in the Grimsel migration shear zone, Paul Scherrer Institut, CH-5232 Villigen PSI (Switzerland).

Kulasiri, D., 2013. Non-fickian Solute Transport in Porous Media: A Mechanistic and Stochastic Theory. Springer.

Le Borgne, T., Dentz, M., Carrera, J., 2008. Lagrangian statistical model for transport in highly heterogeneous velocity fields. Physical review letters, 101(9): 090601.

Le Borgne, T., Gouze, P., 2008. Non - Fickian dispersion in porous media: 2. Model validation from measurements at different scales. Water Resources Research, 44(6).

Levchuk, S., Kashparov, V., Maloshtan, I., Yoschenko, V., Van Meir, N., 2012. Migration of transuranic elements in groundwater from the near-surface radioactive waste site. Applied Geochemistry, 27(7): 1339-1347.

Li, N., Ren, L., 2009. Application of continuous time random walk theory to nonequilibrium transport in soil. Journal of contaminant hydrology, 108(3): 134-151.

Maderich, V. et al., 2014. Dispersion and fate of $90 \mathrm{Sr}$ in the Northwestern Pacific and adjacent seas: Global fallout and the Fukushima Dai-ichi accident. Science of The Total Environment, 494: 261-271.

Margolin, G., Dentz, M., Berkowitz, B., 2003. Continuous time random walk and multirate mass transfer modeling of sorption. Chemical physics, 295(1): 71-80.

Miller, W.M., Chapman, N., McKinley, I., Alexander, R., Smellie, J., 1994. Natural analogue studies in the geological disposal of radioactive wastes. Elsevier.

Mohamed, M., Hatfield, K., Hassan, A., Klammler, H., 2010. Stochastic evaluation of subsurface contaminant discharges under physical, chemical, and biological heterogeneities. Advances in Water Resources, 33(7): 801-812.

Neuman, S.P., Tartakovsky, D.M., 2009. Perspective on theories of non-Fickian transport in heterogeneous media. Advances in Water Resources, 32(5): 670-680. 
Qian, T., Li, S., Ding, Q., Wu, G., Zhao, D., 2009. Two-dimensional numerical modeling of $90 \mathrm{Sr}$ transport in an unsaturated Chinese loess under artificial sprinkling. Journal of environmental radioactivity, 100(5): 422-428.

Rhodes, M.E., Bijeljic, B., Blunt, M.J., 2008. Pore-to-field simulation of single-phase transport using continuous time random walks. Advances in water resources, 31(12): 1527-1539.

Rod, K.A., Um, W., Flury, M., 2010. Transport of strontium and cesium in simulated Hanford tank waste leachate through quartz sand under saturated and unsaturated flow. Environmental science \& technology, 44(21): 8089-8094.

Rubin, S., Dror, I., Berkowitz, B., 2012. Experimental and modeling analysis of coupled non-Fickian transport and sorption in natural soils. Journal of contaminant hydrology, 132: 28-36.

Rumynin, V.G., 2011. Subsurface Solute Transport Models and Case Histories: With Applications to Radionuclide Migration, 25. Springer Science \& Business Media.

Scher, H., Lax, M., 1973. Stochastic transport in a disordered solid. I. Theory. Physical Review B, 7(10): 4491.

Šimůnek, J., van Genuchten, M.T., 2008. Modeling nonequilibrium flow and transport processes using HYDRUS. Vadose Zone Journal, 7(2): 782-797.

Šimůnek, J., van Genuchten, M.T., Šejna, M., 2008. Development and applications of the HYDRUS and STANMOD software packages and related codes. Vadose Zone Journal, 7(2): 587-600.

Šimůnek, J., Van Genuchten, M.T., Šejna, M., Toride, N., Leij, F., 1999. The STANMOD computer software for evaluating solute transport in porous media using analytical solutions of convection-dispersion equation. Versions 1.0 and 2.0. International Ground Water Modeling Center.

Srinivasan, P., Sarmah, A.K., 2014. Assessing the sorption and leaching behaviour of three sulfonamides in pasture soils through batch and column studies. Science of The Total Environment, 493: 535-543.

Swanson, R.D. et al., 2015. Anomalous solute transport in saturated porous media: Relating transport model parameters to electrical and nuclear magnetic resonance properties. Water Resources Research, 51(2): 1264-1283.

Toride, N., Leij, F., Van Genuchten, M.T., 1995. The CXTFIT code for estimating transport parameters from laboratory and field tracer experiments.

Volkova, E., 2009. Sensitivity analysis of the numerical simulation of 90Sr transport for old repositories at the Russian Science Center Kurchatov Institute. Atomic energy, 106(4): 287-293.

Wang, L., Cardenas, M.B., 2014. Non - Fickian transport through two - dimensional rough fractures: Assessment and prediction. Water Resources Research, 50(2): 871-884.

Yin, J., Jeen, S.-W., Lee, D.R., Mayer, K.U., 2014. Reactive transport modeling of $90 \mathrm{Sr}$ sorption in reactive sandpacks. Journal of hazardous materials, 280: 685-695. 


\begin{tabular}{|c|c|c|}
\hline Value & Characteristics of parameters & Transport feature \\
\hline $0<\beta<1$ & $\begin{array}{l}\text { The first and the second moment of the } \psi(t) \text { does } \\
\text { not exist; both transport velocity and dispersion } \\
\text { coefficient vary with time as power laws. }\end{array}$ & $\begin{array}{l}\text { Indicates a highly non-Fickian } \\
\text { dispersion transport. }\end{array}$ \\
\hline $1<\beta<2$ & $\begin{array}{l}\text { The second moment of the } \psi(t) \text { does not exist, } \\
\text { where transport velocity is constant but dispersion } \\
\text { coefficient still vary with time. }\end{array}$ & $\begin{array}{l}\text { Presents moderate non-Fickian } \\
\text { transport or moderately } \\
\text { dispersive systems. }\end{array}$ \\
\hline$\beta \geqslant 2$ & $\begin{array}{l}\text { The first and second moments of the } \psi(t) \text { exist, both } \\
\text { transport velocity and dispersion coefficient are } \\
\text { constant. }\end{array}$ & $\begin{array}{l}\text { Displays Fickian transport, } \\
\text { Gaussian distributions of plume }\end{array}$ \\
\hline
\end{tabular}

Table 2 Fitted values of parameters of strontium for ADE and CTRW

\begin{tabular}{|c|c|c|c|c|c|c|c|c|c|c|}
\hline & \multicolumn{3}{|c|}{ ADE (CXTFIT) } & \multicolumn{7}{|c|}{ CTRW (MATLAB toolbox) } \\
\hline & $\begin{array}{c}v^{I} \\
(\mathrm{~cm} / \mathrm{d})\end{array}$ & $\begin{array}{c}D^{I} \\
\left(\mathrm{~cm}^{2} / \mathrm{d}\right)\end{array}$ & $R^{l}$ & $\begin{array}{c}v_{\psi} \\
(\mathrm{cm} / \mathrm{d})\end{array}$ & $\begin{array}{c}D_{\psi} \\
\left(\mathrm{cm}^{2} / \mathrm{d}\right)\end{array}$ & $\beta$ & $t_{1}(\mathrm{~d})$ & $t_{2}(\mathrm{~d})$ & $\Lambda$ & $\mu$ \\
\hline $\mathrm{a}$ & $79.78 \pm 5.78$ & $286.56 \pm 1.44$ & $0.997 \pm 0.111$ & 55.34 & 178.56 & 1.80 & $1.48 \mathrm{E}-2$ & 1.09 & 0.05 & 0.01 \\
\hline $\mathrm{b}$ & $46.30 \pm 3.22$ & $67.25 \pm 15.50$ & $1.017 \pm 0.069$ & 11.23 & 30.65 & 2.16 & 4.32E-2 & 5.93 & 0.04 & 0.01 \\
\hline $\mathrm{c}$ & $22.50 \pm 0.59$ & $11.10 \pm 2.20$ & $1.039 \pm 0.027$ & 10.8 & 3.44 & 2.27 & $4.25 \mathrm{E}-2$ & 4.60 & 0.03 & 0.03 \\
\hline $\mathrm{d}$ & $9.21 \pm 9.20$ & $22.37 \pm 4.79$ & $0.999 \pm 0.111$ & 20.25 & 215.94 & 0.31 & $2.05 \mathrm{E}-2$ & $4.06 \mathrm{E} 3$ & 0.09 & 0.08 \\
\hline $\mathrm{e}$ & $1.05 \pm 0.01$ & $0.57 \pm 0.03$ & $1.053 \pm 0.027$ & 0.52 & 0.12 & 1.43 & $2.53 \mathrm{E}-1$ & $3.62 \mathrm{E} 3$ & 0.12 & 0.12 \\
\hline $\mathrm{f}$ & $0.83 \pm 0.01$ & $0.55 \pm 0.04$ & $1.076 \pm 0.048$ & 0.47 & 0.10 & 1.26 & $3.69 \mathrm{E}-1$ & $6.59 \mathrm{E} 3$ & 0.13 & 0.09 \\
\hline $\mathrm{g}$ & $0.67 \pm 0.01$ & $0.47 \pm 0.04$ & $1.093 \pm 0.029$ & 0.38 & 0.12 & 0.62 & $6.24 \mathrm{E}-1$ & $6.89 \mathrm{E} 3$ & 0.15 & 0.15 \\
\hline $\mathrm{h}$ & $0.58 \pm 0.01$ & $0.43 \pm 0.06$ & $1.194 \pm 0.017$ & 0.31 & 0.03 & 0.87 & $9.11 \mathrm{E}-1$ & $1.07 \mathrm{E} 4$ & 0.18 & 0.16 \\
\hline
\end{tabular}

667 Columns a-d: packed by sand, with sprinkling volumetric flow rate $Q=100,50,20,2 \mathrm{~mL} / \mathrm{d}$, 668 respectively; Columns e-h: $Q=2 \mathrm{~mL} / \mathrm{d}$, packed by sand+clay (3:1), sand+clay $(1: 1)$, sand+clay (1:3), 669 clay, respectively. Note that $\mathrm{Sr}^{2+}$ transport velocities can be calculated by dividing $v$ by $R$ based on 670 the Eq. (1). In the ADE model, the fitted $v$ represents the average pore water velocity. In the CTRW 671 framework, $v_{\psi}$ directly refers to tracer transport velocity.

$672{ }^{1}$ : The parameters values with $95 \%$ confidence limits.

Table 3 Estimated values of Peclet number for the columns

\begin{tabular}{lllllllll}
\hline column & $\mathrm{a}$ & $\mathrm{b}$ & $\mathrm{c}$ & $\mathrm{d}$ & $\mathrm{e}$ & $\mathrm{f}$ & $\mathrm{g}$ & $\mathrm{h}$ \\
\hline$P e^{I}$ & $4.6 \sim 5.6$ & $9.4 \sim 17.2$ & $29.6 \sim 56.1$ & $0.01 \sim 18.8$ & $31.2 \sim 78$ & $25.1 \sim 84.6$ & $23.3 \sim 57$ & $20.9 \sim 186$ \\
\hline${ }^{I}$ : The values were estimated using the inverse modelling results listed in Table 2.
\end{tabular}




\section{Figure captions}

Fig. 1. Schematic illustration of the experimental setup

Fig. 2. Simulated leach concentrations of $\mathrm{Br}^{-}$(a) and $\mathrm{Sr}^{2+}$ (b) over column experimental duration under different sprinkling volumetric flow rate $(Q)$ conditions according to equations (2 3).

Fig. 3. Observed cumulative mass fraction of $\mathrm{Br}^{-}(\mathrm{a})$ and $\mathrm{Sr}^{2+}$ (b) eluted from corresponding columns, calculated by Eq. (12). Columns a-d: packed by sand, sprinkling volume flow rate $Q=100,50,20,2$ $\mathrm{mL} / \mathrm{d}$, respectively; Columns e-h: $Q=2 \mathrm{~mL} / \mathrm{d}$, packed by sand and clay (3:1), sand and clay (1:1), sand and clay (1:3), clay, respectively.

Fig. 4. Measured and best-fitted BTCs of $\mathrm{Sr}^{2+}$, using ADE/CTRW for column experiments. The corresponding fitted values of parameters for the columns are shown in Table 2. The first four columns (a-d) are packed with sand, with sprinkling volume flow rate $Q=100,50,20,2 \mathrm{~mL} / \mathrm{d}$, respectively; the next three columns (e-h) are packed with different ratios of sand to clay: e 3:1, f 1:1, g 1:3, respectively. The column-h was packed with clay. Note that columns e-h are used by applying the same volumetric flow rate $2 \mathrm{~mL} / \mathrm{d}$. Dashed line is the equilibrium ADE model fit and solid line is the CTRW-based TPL model fit. 
Fig. 1

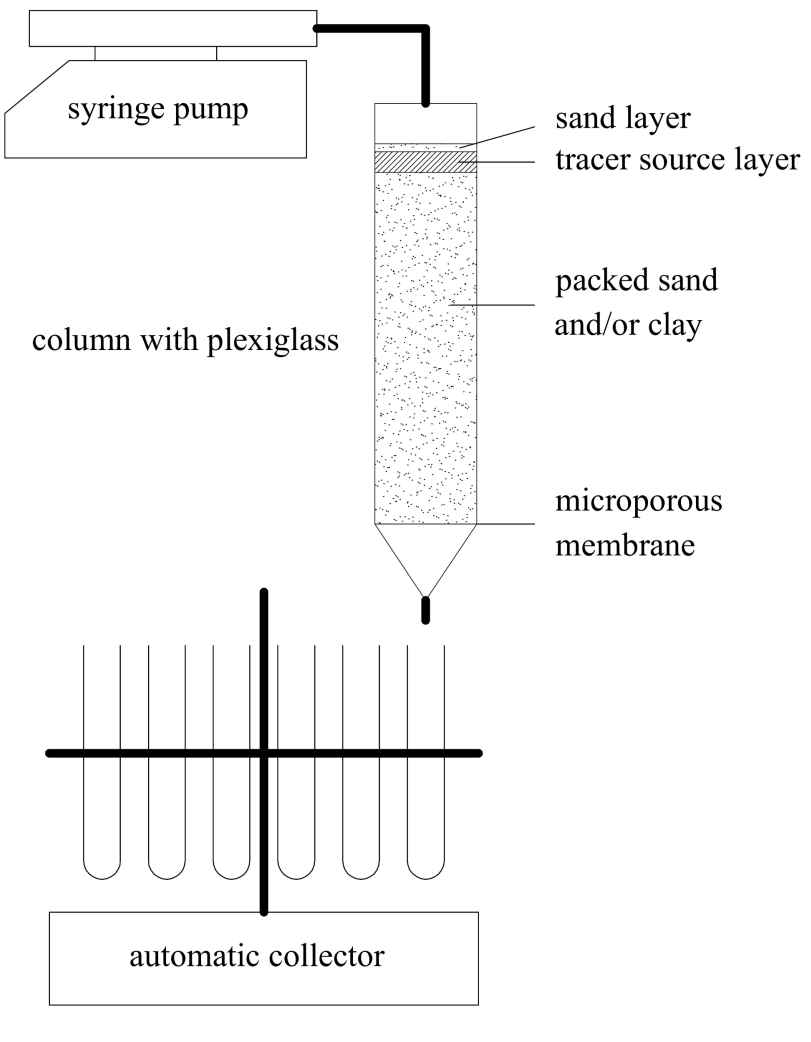


Fig. 2

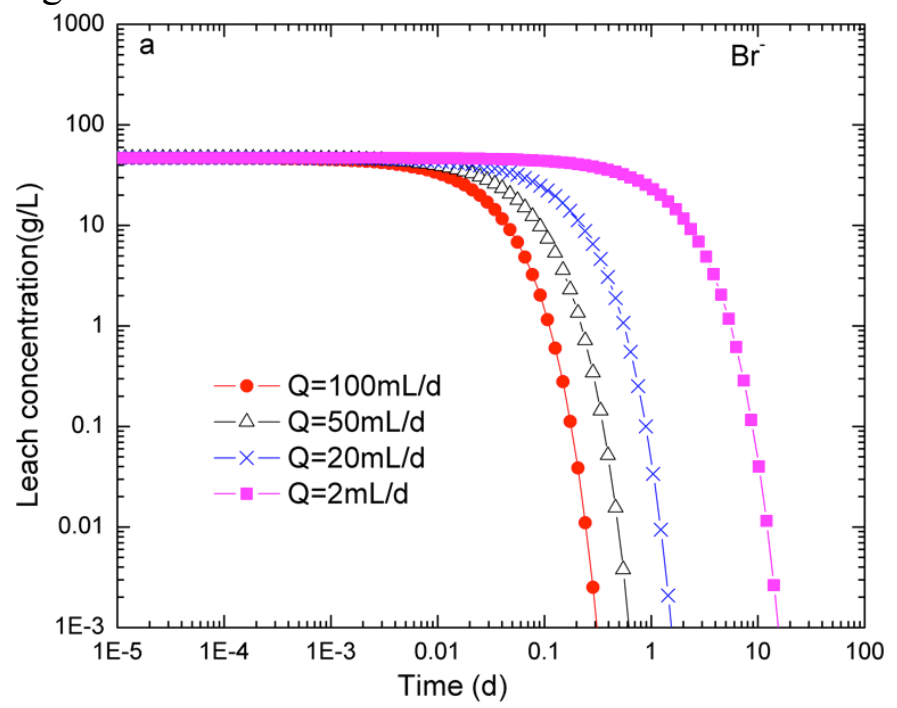

704

705

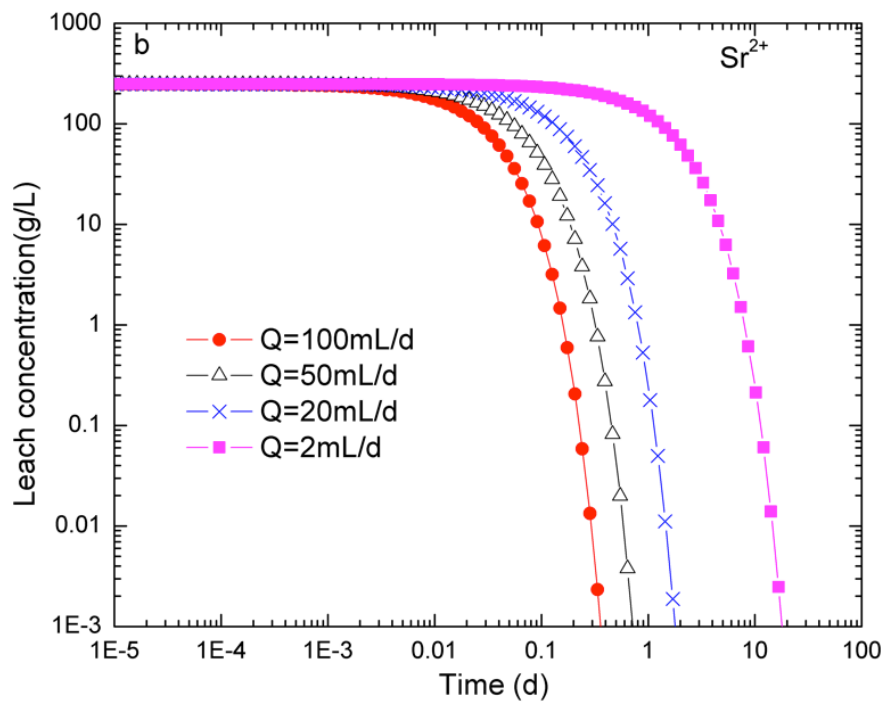


Fig. 3
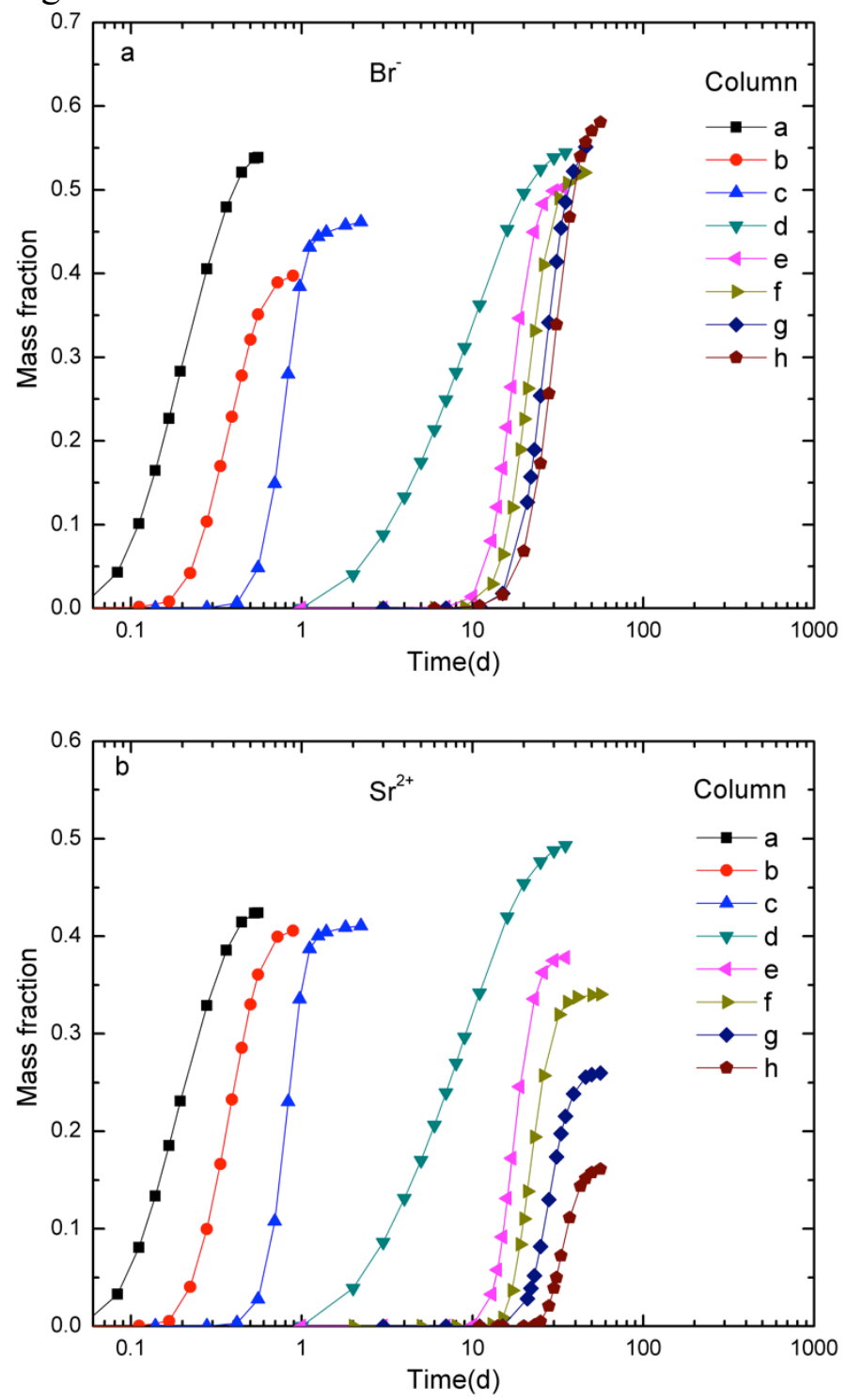
$713 \quad$ Fig. 4

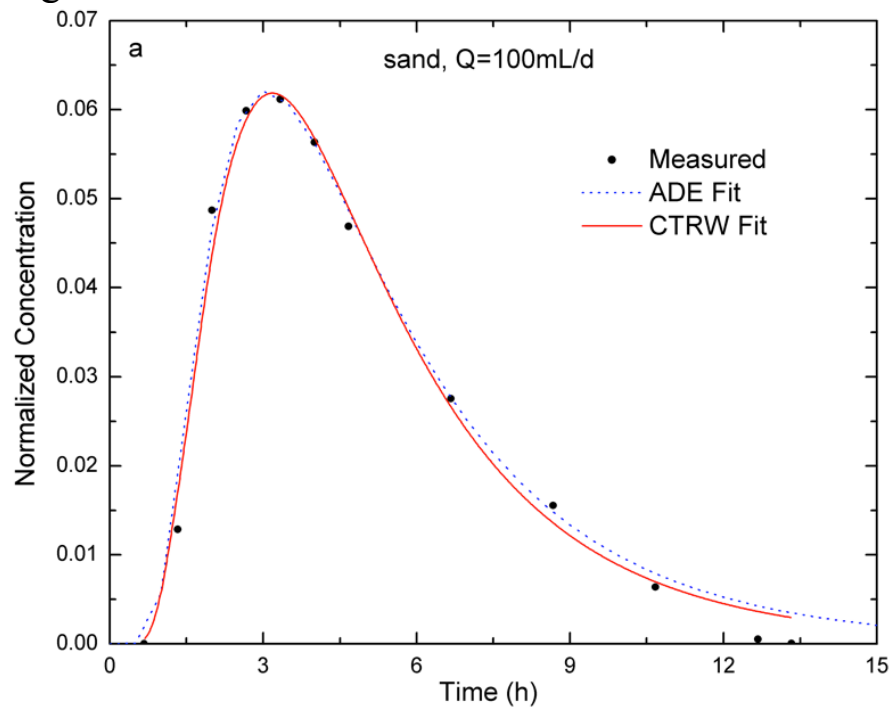

714

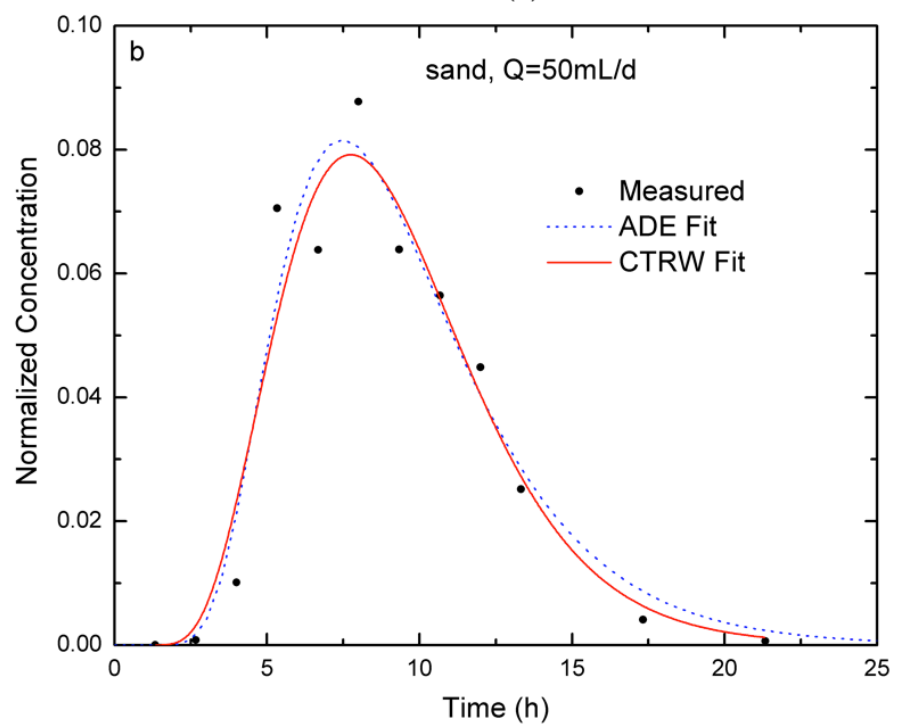

715

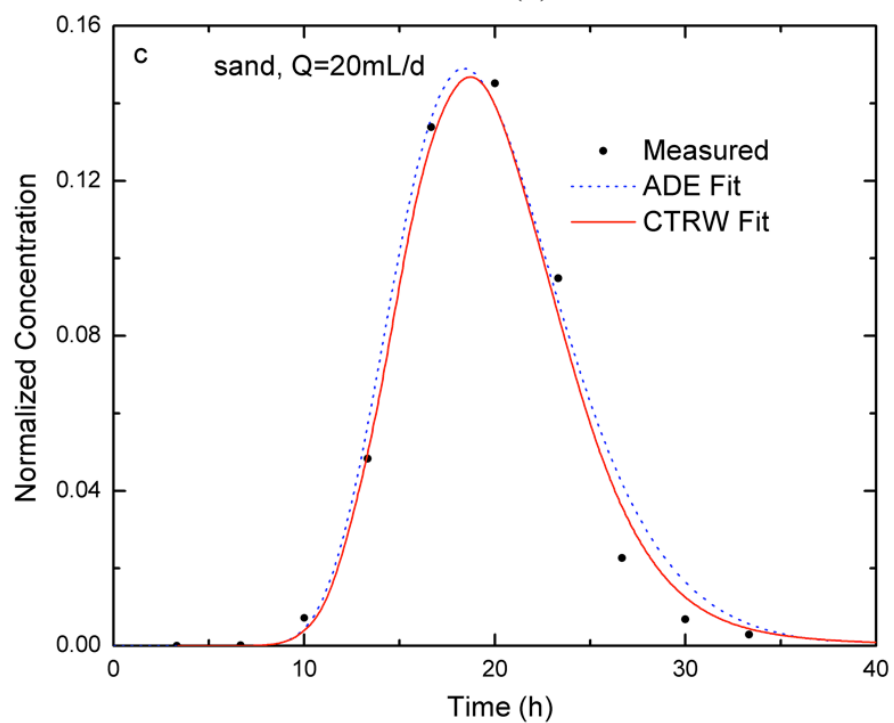




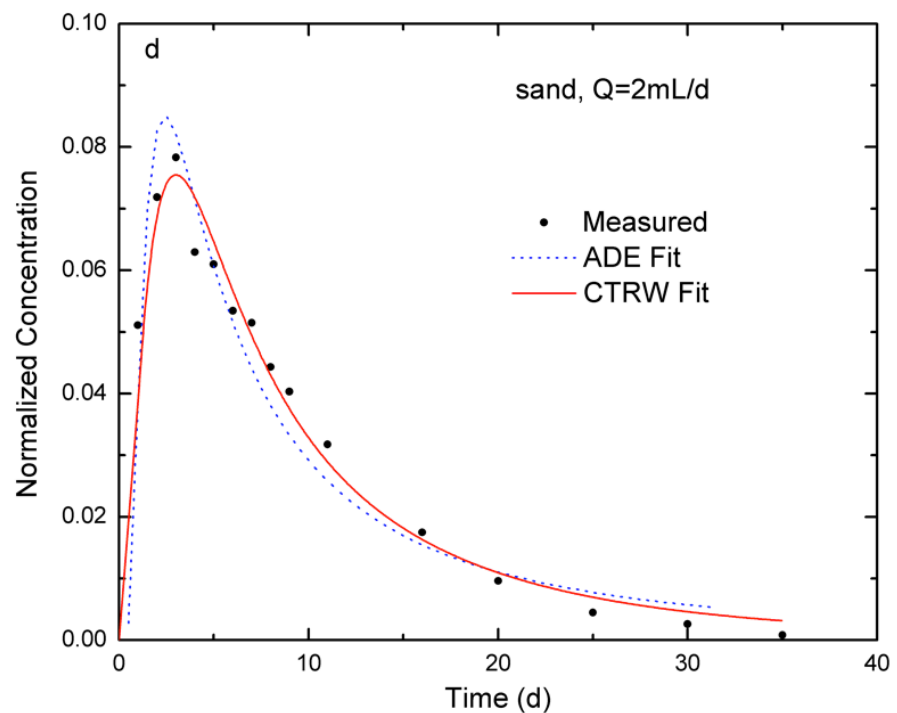

717

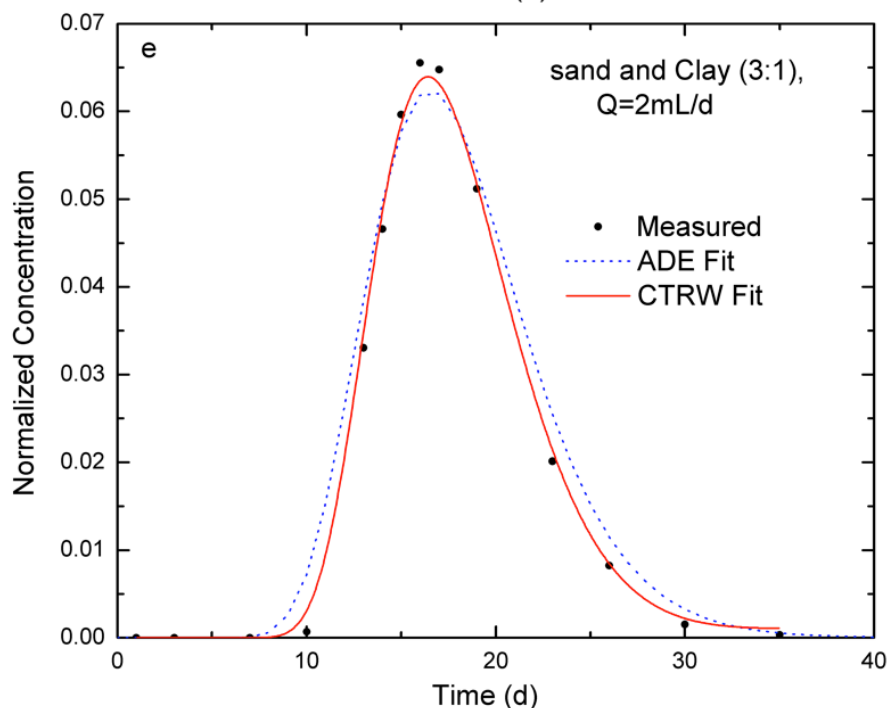

718

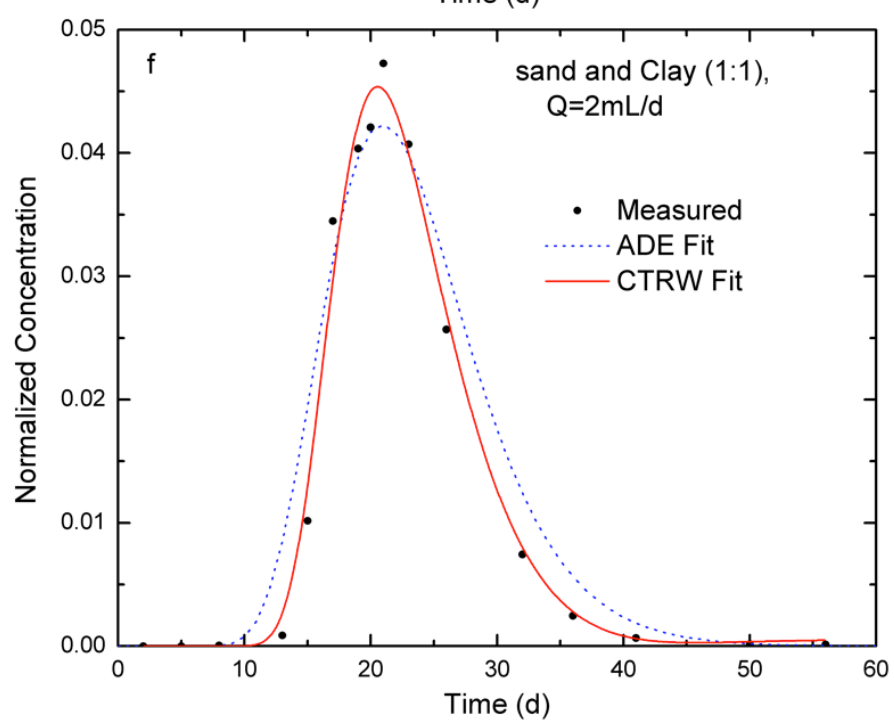




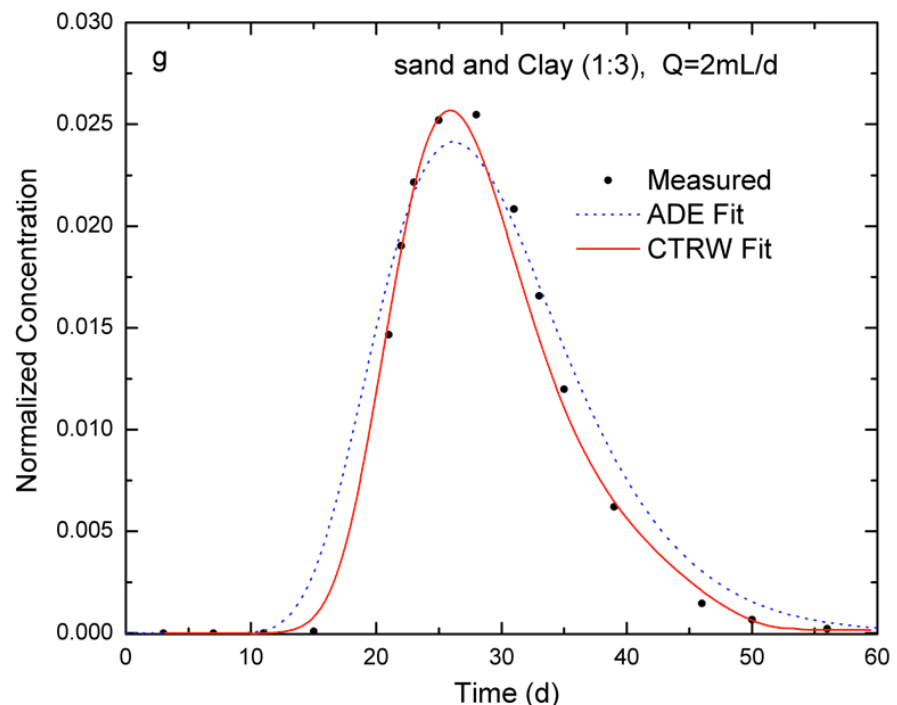

720

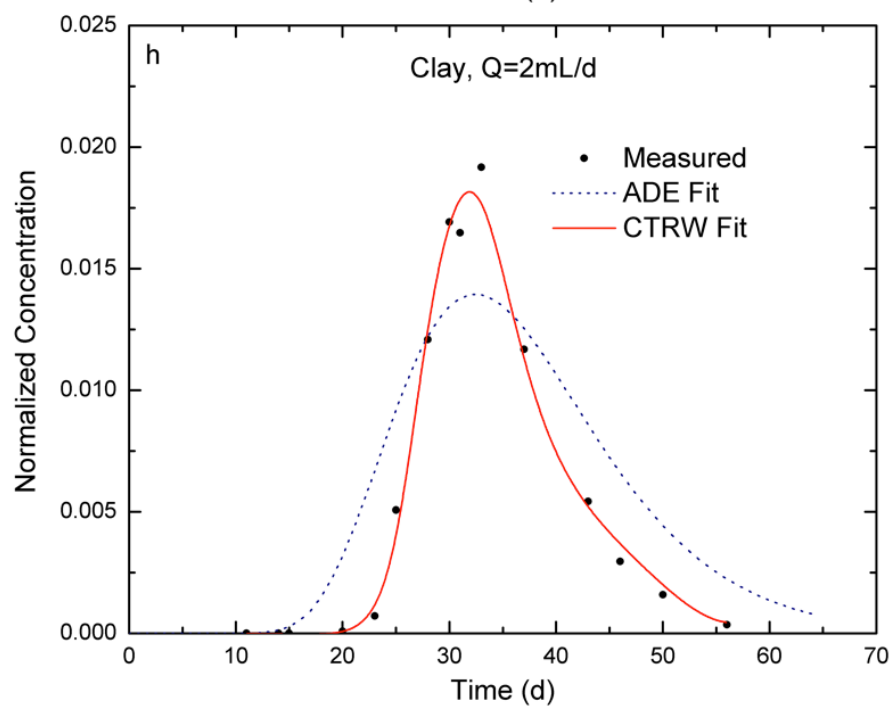

